\title{
GLAD!
}

Revue sur le langage, le genre, les sexualités

$10 \mid 2021$

Varia

\section{Alain Rabatel et Laurence Rosier (dir.). 2019. « Les défis de l'écriture inclusive ", Le discours et la langue 11(1)}

\section{Éléonore de Beaumont}

\section{(2) OpenEdition}

Journals

Édition électronique

URL : https://journals.openedition.org/glad/2654

DOI : $10.4000 /$ glad.2654

ISSN : 2551-0819

Éditeur

Association GSL

\section{Référence électronique}

Éléonore de Beaumont, «Alain Rabatel et Laurence Rosier (dir.). 2019. «Les défis de l'écriture inclusive », Le discours et la langue 11(1) », GLAD! [En ligne], 10 | 2021, mis en ligne le 15 juillet 2021, consulté le 26 juillet 2021. URL : http://journals.openedition.org/glad/2654 ; DOI : https://doi.org/ $10.4000 /$ glad.2654

Ce document a été généré automatiquement le 26 juillet 2021.

\section{(c) (i) (9)}

La revue GLAD! est mise à disposition selon les termes de la Licence Creative Commons Attribution Pas d'Utilisation Commerciale - Pas de Modification 4.0 International. 


\title{
Alain Rabatel et Laurence Rosier (dir.). 2019. « Les défis de l'écriture inclusive ", Le discours et la langue 11(1)
}

\author{
Éléonore de Beaumont
}

\section{RÉFÉRENCE}

Alain Rabatel et Laurence Rosier (dir.). 2019. « Les défis de l'écriture inclusive », Le discours et la langue 11(1)

1 Sous la coordination d'Alain Rabatel (professeur à l'Université Lyon 1, laboratoire ICAR) et de Laurence Rosier (Université libre de Belgique, laboratoire Ladisco/striges), dix chercheurs et chercheuses s'attèlent à l'analyse des «défis » du langage non sexiste. Dès l'introduction, l'écriture inclusive est présentée comme un événement linguistique (au sens de Guilhaumou 1996), qu'il est donc nécessaire d'appréhender d'un point de vue historique, et comme un événement de la réception (Calabrese 2018), ce qui implique que les réactions des locuteurs et locutrices font partie intégrante de l'analyse de l'objet. Les huit articles qui composent cette publication permettent de prendre de la distance avec les polémiques suscitées depuis $2017^{1}$ par ces nouvelles modalités langagières, et invitent à une réflexion historique et épistémologique.

2 L'expression «écriture inclusive » est entrée récemment dans le vocabulaire commun ${ }^{2}$, mais plusieurs articles s'attachent à la replacer dans l'histoire des revendications pour la féminisation ou "démasculinisation» (Viennot 2014) de la langue. Dès l'introduction, Alain Rabatel et Laurence Rosier rappellent que les liens entre féminisme et réflexion linguistique étaient déjà au cœur de l'ouvrage de Marina Yaguello, Les mots et les femmes, en 1978. Bernard Cerquiglini consacre un long développement à l'émergence de cette évolution linguistique dans la francophonie, y 
voyant un signe de l'émancipation de la langue par rapport à la norme française. Dans son article, Laurence Rosier rend hommage (on pourrait parler de femmage 3 ) à AnneMarie Houdebine pour son travail sur la norme, et Alpheratz rappelle les travaux fondateurs de Claire Michard (2012) et de Lucy Michel (2016). Ce numéro s'inscrit donc à la fois dans cette histoire vieille déjà de plus de quarante ans, mais aussi dans la contemporanéité des débats liés au "psychodrame » (Cerquiglini 2018) de l'émergence du point médian, la technique linguistique la plus controversée du langage non sexiste. L'amplitude géographique des contributeur/trices en fait également sa richesse: les contributions de linguistes françaises, belges et suisse ainsi que la dimension interculturelle de l'article de Laure Gardelle, permettent un élargissement du regard, nécessaire pour aborder cet objet d'étude pleinement francophone qu'est le langage inclusif.

Ce numéro est placé sous le signe de la diversité. Diversité des enjeux abordés, tout d'abord: Jean-Marie Klinkenberg (professeur émérite de l'Université de Liège et président du Conseil de la langue française et de la politique linguistique de la Fédération Wallonie-Bruxelles) insiste dans le premier article sur la nécessité d'aborder l'écriture inclusive d'un point de vue social et politique, et pas seulement linguistique. Selon lui, les techniques (lexicales et syntaxiques) langagières ne sont qu'un moyen, parmi d'autres, d'assurer la visibilisation des femmes dans la société, mais un moyen indispensable dans le combat pour l'égalité. Il défend toutefois une position mesurée vis-à-vis de l'écriture inclusive en mettant en garde contre les exclusions qu'elle pourrait susciter.

4 Les trois articles suivants proposent une réflexion sur la norme et les instances normatives: Bernard Cerquiglini (Université Paris-Diderot) décrypte la stratégie de l'Académie française pour conserver ses prérogatives sur la langue afin d'expliquer le « retard hexagonal» (p. 31) par rapport à d'autres pays francophones (Canada, Suisse, Belgique) concernant la féminisation des noms de métiers, titres et fonctions. Laurence Rosier, ensuite, dans son analyse de la violence verbale lors des débats sur la langue, prend position contre le dénigrement de la parole populaire réduite à la polémique, quand les linguistes participeraient à des controverses: elle considère que «la posture consistant à retirer l'analyse scientifique de l'arène publique reproduit une vision dominante de la langue légitime » (p. 44). Enfin, Alpheratz (Paris-Sorbonne Université, laboratoire STIH) propose une synthèse rigoureuse de la conceptualisation du français inclusif et analyse le processus de "grammatisation $»^{4}$ (p. 54) à l'œuvre pour faire passer le français inclusif du discours (ici, les usages particuliers) à la langue (ici, le système propre à une communauté linguistique).

5 Puis, deux articles s'intéressent à l'existence du neutre dans la langue française : Dan Van Raemdonck (Université libre de Bruxelles, laboratoire Ladisco/Tradital/CLIN) et Patrick Charaudeau (Université de Paris XIII, laboratoire CNRS/Lcp/Irisso) récusent les accusations de sexisme faites au français en défendant l'idée que seuls les discours portés sur la langue peuvent être sexistes. Ils proposent chacun une analyse du neutre en français : dans une approche morphosyntaxique et s'appuyant sur les mécanismes diachroniques, Raemdonck défend l'adoption d'une terminologie tripartite ("genre non marqué ", "genre marqué masculin » et "genre marqué féminin »), un discours qui permettrait de ne plus confondre masculin et neutre, alors que Charaudeau s'appuie sur la notion de neutralisation discursive pour nuancer la pertinence des modalités inclusives en contexte. Pour contrebalancer ces deux points de vue, 
l'approche psycholinguistique de Pascal Gygax aurait pu être intéressante: ses expériences, notamment auprès des enfants, montrent que les emplois génériques du masculin induisent des représentations mentales biaisées, favorables au masculin.

Enfin, les deux derniers articles décentrent l'analyse en comparant les stratégies adoptées dans le monde francophone à celles d'autres aires linguistiques. Tout d'abord, Daniel Elmiger (Université de Genève), Eva Schaffer-Lacroix (Sorbonne Université) et Verena Tunger (Zurich) présentent un projet d'étude sur les évolutions des pratiques effectives du personnel administratif suisse, c'est-à-dire en contexte décentralisé et plurilingue (puisque la Suisse compte quatre langues nationales: le français, le romanche, l'allemand et l'italien). Leur enquête s'appuie sur la méthode de la linguistique de corpus, avec une analyse d'occurrences dans les textes administratifs de 1849 à 2014, ainsi que sur des entretiens visant à recueillir l'opinion du personnel. Il en ressort que les pratiques de l'administration francophone ont peu évolué, contrairement à celles de son pendant germanophone. Enfin, Laure Gardelle (Université Grenoble Alpes) propose une comparaison éclairante des stratégies inclusives anglophones et francophones : elle analyse le succès des premières et l'échec des secondes en s'appuyant sur les systèmes linguistiques. Elle montre ainsi que les obstacles cognitifs peuvent être plus puissants que la pertinence sociologique d'une proposition. L'intérêt de cet article réside également dans son approche pragmatique, puisque la chercheuse propose plusieurs réécritures de textes, notamment un extrait de rapport de jury de concours, dans un langage épicène : elle défend ainsi une position intermédiaire, mettant en avant la nécessité sociale d'un changement des pratiques linguistiques mais aussi le coût cognitif de certaines modalités du langage non sexiste, comme les doublets et les points médians.

7 Les contributeurs et contributrices de ce numéro défendent des positions souvent différentes, voire clairement opposées, ce qui n'apparaissait pas de manière évidente à la lecture de l'introduction. Ainsi, pour Laure Gardelle «il est bien établi aujourd'hui que genre non marqué morphologiquement ne signifie pas réelle indifférenciation des sexes» (p. 157) et pour Jean-Marie Klinkenberg, les formes masculines sont «fallacieusement réputées neutres » (p. 18). Au contraire, Dan Van Raemdonck propose de parler d'un "genre non marqué", et Patrick Charaudeau soutient un emploi parcimonieux du masculin générique. Les différents articles font apparaitre en creux les controverses qui agitent l'analyse du genre grammatical : existe-t-il un genre neutre (ou «non marqué ») en français? Peut-on en créer un ou le système linguistique français est-il irrémédiablement binaire ? Doit-on considérer que les substantifs ont un genre qui leur est propre ou que le féminin est dérivé du masculin? Autant de points d'achoppement qui distinguent les orientations théoriques des auteurs et autrices de ce numéro.

De façon à la fois surprenante et extrêmement intéressante, la dimension militante de l'objet étudié a poussé plusieurs contributeurs et contributrices à expliciter leur positionnement en tant que linguiste. Ainsi, Dan Van Raemdonck se définit tout d'abord en tant que morpho-syntacticien, mais il explique dans une note qu'il « troque ici, pour la première fois, le nous dit scientifique, pour un je » (p. 76) et se présente d'un point de vue social comme homme blanc, homosexuel, juif, etc. Paradoxalement, il souligne plus loin la nécessité d'«agir en scientifique » et le danger du militantisme dans la recherche : porter un jugement de valeur sur la langue l'exposerait au risque de «tourner puriste normativiste» (p. 77). Laure Gardelle tient également une posture 
paradoxale : elle précise d'abord qu'il s'agit « le plus indépendamment possible de toute implication idéologique, [...] d'examiner les conséquences sur le fonctionnement interne de la langue des ajustements lexicaux et/ou grammaticaux proposés » (p. 154). Toutefois, elle s'interroge: «[C]ertaines préconisations peuvent-elles être mises en avant pour qui voudrait intervenir? » (p. 154, nous soulignons). Plusieurs contributeurs et contributrices insistent sur l'importance de prendre en compte l'usage de la communauté linguistique pour les évolutions langagières. On peut néanmoins s'interroger sur l'impact du discours des linguistes sur les opinions des locuteurs et locutrices. Alpheratz, par exemple, assume la dimension transformatoire du travail du grammairian ${ }^{5}: \mathrm{al}^{6}$ note que l'entreprise d'outillage métalinguistique du langage inclusif pourrait faire sortir ce dernier de l'agrammaticalité et le présenter comme légitime. Est-il alors possible pour les linguistes d'avoir une posture réellement neutre vis-à-vis de l'écriture inclusive ? Est-ce même souhaitable ? Les fondatrices de GLAD! rappelaient dans l'introduction du premier numéro (Abbou et alii 2016) la nécessité de réfléchir au caractère situé de la production des savoirs et soulignaient à quel point les études de genre et les études féministes permettaient de « revisiter les discours à la lumière de l'idéologie et des rapports sociaux » $(2016: 2)$.

9 Exceptéee Alpheratz, les linguistes qui ont contribué à cette revue adoptent une position plutôt mesurée vis-à-vis du langage inclusif. On pourrait regretter que des chercheureuses soutenant des positions plus tranchées n'aient pas participé à l'élaboration de ce numéro, comme Julie Abbou qui soutient une approche émancipatrice plutôt qu'inclusive : selon elle, puisque le langage non sexiste est de plus en plus accepté et utilisé à des fins communicationnelles, il s'agit de chercher à bousculer, à déranger, à créer de l'illisible pour viser une pratique véritablement politique de la grammaire et sortir de l'état de minorité. En outre, la question de la création et de l'institutionnalisation d'un genre neutre, développée par Alpheratz semble avoir déjà évolué depuis la parution de la revue début 2019 et tient sans doute aujourd'hui une place plus importante qu'alors. D'autres propositions, comme le féminin générique, ont notamment été analysées récemment par Daniel Elmiger (2020) et sont utilisées par des chercheuses comme Maria Candea. Néanmoins, la diversité des méthodes et des approches théoriques ainsi que l'inscription de ces contributions dans un espace francophone élargi font de cette revue une lecture riche, tant sur les plans historique et épistémologique que pragmatique.

\section{BIBLIOGRAPHIE}

ABBOU Julie, CANDEA Maria, COUTANT Alice, GÉRARDIN-LAVERGE Mona, KATSIKI Stavroula, MARIGNIER Noémie, MICHEL Lucy, THÉVENET Charlotte. 2016. « GLAD! revue féministe et indisciplinée », GLAD!, 1. URL : http://journals.openedition.org/glad/260

CALABRESE Laura. 2017. « L'évènement de réception. Un évènement de parole du côté des publics » Sciences de la société, 101. URL : https://journals.openedition.org/sds/7109

CERQUIGLINI Bernard. 2018. Le ministre est enceinte, Paris : Seuil. 
ELMIGER Daniel, 2020, « Les genres récrits : chronique n7 », GLAD!, 9. URL : https:// journals.openedition.org/glad/2346

GUILHAUMOU Jacques. 1996. « Vers une histoire des évènements linguistiques. Un nouveau protocole d'accord entre l'historien et le linguiste » Histoire, épistémologie, langage, 18/2 :103-126.

MICHARD Claire. 2012. « Rapport de sexage, effet idéologique et notion de sexe en français », $L a$ face cachée du genre : Langage et pouvoir des normes, Paris : Presses Sorbonne Nouvelle. URL : https://books.openedition.org/psn/3113?lang=fr

MICHEL Lucie. 2016. La relation entre genre grammatical et dénomination de la personne en langue française, thèse en Linguistique, Dijon : Université de Bourgogne.

VIENNOT, Éliane. 2014. Non, le masculin ne l'emporte pas sur le féminin, Petite histoire des résistances de la langue française, Donnemarie-Dontilly : iXe.

YAGUELLO Marina. 1978. Les mots et les femmes, Paris : Payot.

\section{NOTES}

1. En 2017, la parution du manuel Questionner le monde, niveau CE2, chez Hatier, a suscité la polémique pour son utilisation de l'écriture inclusive. La maison d'édition a déclaré ensuite avoir appliqué les recommandations du Haut Conseil à l'Égalité, parues en 2016 et qui n'avaient pas fait de bruit à l'époque.

2. Julie Abbou travaille actuellement sur les origines de la terminologie «écriture inclusive » : elle montre qu'il s'agit d'une notion issue de la théologie protestante états-unienne des années 1980 (Abbou J. « «Pratiquer une politique de la grammaire : de la bousculade à l'inclusion (et retour)", Séminaire Genre, Médias et Communication, 20 mars 2020). La première occurrence d'origine non-théologienne de l'expression remonte à 1992 (Dumais, H. 1992. "Pour un genre à part entière ", Recherches féministes 5 (1), p. 169-174) mais elle ne s'est véritablement répandue auprès du grand public qu'en 2017, à l'occasion de la polémique suscitée par la publication du manuel Hatier.

3. Néologisme proposé par des associations féministes comme Osez le féminisme! et défendu par des artistes comme Typhaine D ou des linguistes comme Laélia Véron, il signifie «hommage rendu à une femme ». Il permet d'attirer l'attention sur l'omniprésence du masculin dans le lexique.

4. Défini par Neveu comme le «processus selon lequel une notion grammaticale intègre un dispositif métalinguistique » (2004:144).

5. Néologisme employé par Alpheratz et défini comme suit : "Proposition de flexion au genre neutre de l'unité lexicale grammairien / grammairienne ». La richesse de ce numéro tient aussi à la diversité des techniques du langage inclusif employées par les contributeurs et contributrices: doublets, doublets abrégés par un point bas (Laurence Rosier), un tiret ou un point médian, néologismes lexicaux (Alpheratz) ou encore langage épicène (Laure Gardelle).

6. «L'identité de genre d'Alpheratz s'exprime par l'usage du pronom al et des accords au neutre » (alpheratz.fr, consulté le 30 mars 2021) 
INDEX

Thèmes : Actualités

Keywords : inclusive writing, non-sexist language, grammatical gender, neutral

Mots-clés : écriture inclusive, langage non sexiste, genre grammatical, neutre

\section{AUTEURS}

\section{ÉLÉONORE DE BEAUMONT}

Éléonore de Beaumont est doctorante en sciences du langage, membre de l'équipe de recherche en didactique des langues et sociolinguistiques de l'ATILF. Sa thèse porte sur l'enseignement du genre grammatical à un public turcophone, dont la langue première n'a pas de genre, dans le cadre du FLE (Français Langue Étrangère). Elle travaille notamment sur les possibilités de l'enseignement d'un langage plus inclusif en classe de FLE et sur ses conséquences en matière de représentations sociolinguistiques des apprenantes. 